\title{
CAVITY-BEAM INSTABILITIES ON THE SRS AT DARESBURY.
}

\section{P A McINTOSH and D M DYKES, Daresbury Laboratory, Daresbury, Warrington WA4 4AD, UK.}

\section{Abstract}

Malfunctions in the water control circuitry on the RF cavities have induced beam movements in both the horizontal and vertical planes which have been observed by users. A possible cause of such movements could be a cavity Higher Order Mode (HOM). The SRS cavity HOM spectra exhibits some Dipole modes which could produce such an effect and investigations have been performed on the SRS which have tried to identify a HOM as the cause of the beam position movement. The data presented here is the result of these investigations, whereby, cavity tuner position, cavity temperature and cavity HOM spectra have been monitored (with beam) as a function of electron beam and photon beam positions.

\section{INTRODUCTION}

As the temperature of the cavity 'cooling' water changes, the physical dimensions of the cavity are altered, and to keep the cavity on tune (the phase of the cavity is kept fixed w.r.t. the phase of the input waveguide) an automatic tuner loop moves a mechanical plunger. This plunger not only changes the frequency of the fundamental mode, but also the HOM's.

Beam movements in the horizontal and vertical planes have been observed and have been attributed to malfunctions in the water control circuitry on cavities 1 and 3 . Undulator beam line station scientists have reported smearing of the undulator output spectrum under these conditions, similar to what has been experienced at ELETTRA[1], a possible cause of such movements could be a cavity HOM.

The effect on the electron beam position of varying the cavity tuner has been monitored using electron beam position monitors (BPM) and tungsten vane photon beam position monitors (TVM).

Preliminary investigations of the SRS cavity HOM spectra indicate that some Dipole modes exist which have comparatively large frequency shifts as a function of tuner position and which act in both planes.

\section{BEAM STUDIES INVESTIGATIONS.}

\section{A. Effect of Cavity Tuner on Electron and Photon BPM's.}

A one minute sawtooth was introduced onto the tuner control position of a cavity and its effect on BPMs and TVMs were recorded. Fig.1 illustrates the behaviour of cavity tuner position and its effect on TVM photon beam position. The $6 \mu \mathrm{m}$ oscillation observed on the TVM was more importantly, observed by station scientists on the beam line.

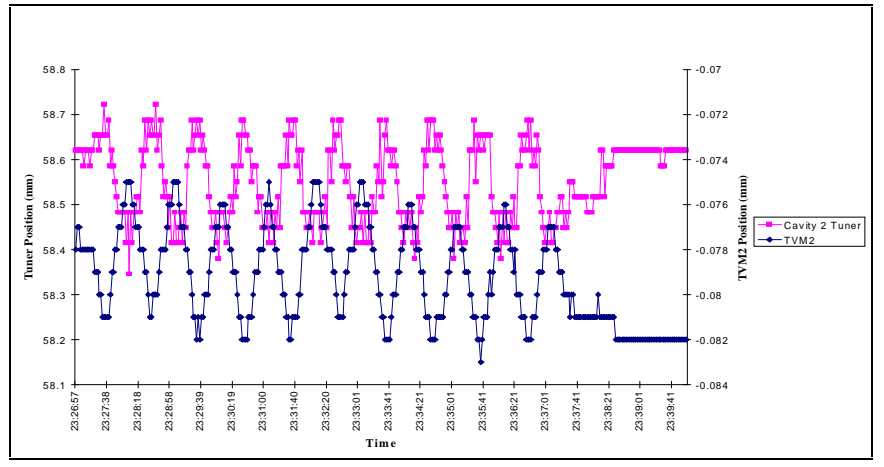

Figure 1. Cavity Tuner Position and Measured Oscillation on TVM.

Fig. 2 is a typical example of the horizontal and vertical beam movements at the BPMs recorded during this experiment.

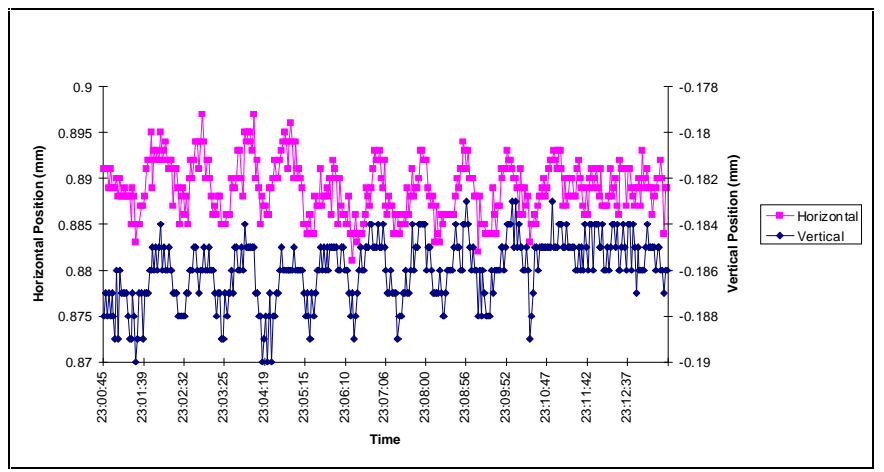

Figure 2. Corresponding Electron Beam Position Movements.

\section{B. The Effect of Varying Cavity Temperature on Electron and Photon BPM's.}

By operating a cavity at a temperature different to nominal, similar observations were made of the BPM and TVM readings. Precise measurements were made of the electron beam output spectrum so that the difference in output spectrum when the cavity was at nominal temperature and when it was at a different temperature, would indicate particular resonances that could be causing the electron beam movements.

The cavity temperature was reduced by $5^{\circ} \mathrm{C}$ and then brought back to nominal operating temperature in $1^{\circ} \mathrm{C}$ increments; at each point recording BPM, TVM and beam spectra. 
Fig.3, shows TVM2 position for a 10 minute period, for each of the cavity temperature settings. The starting point offsets for each cavity temperature plot, is a function of the time needed for the cavity temperature to stabilise. It is not until the cavity temperature reaches $47^{\circ} \mathrm{C}$, that oscillations are apparent in the photon beam position monitor. These oscillations are visible at most of the SRS beam lines and are typically $10-20 \mu \mathrm{m}$ p-p amplitude.

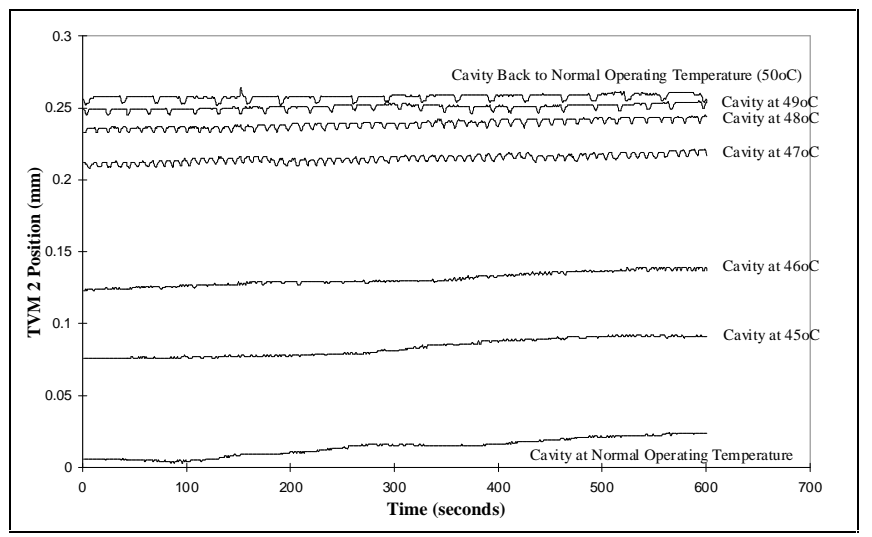

Figure 3. TVM2 Position Readings at Different Cavity Temperatures.

The beam spectra output at this new cavity temperature was compared with the spectra at normal cavity operating temperature and the differences observed in Fig's 4 and 5.

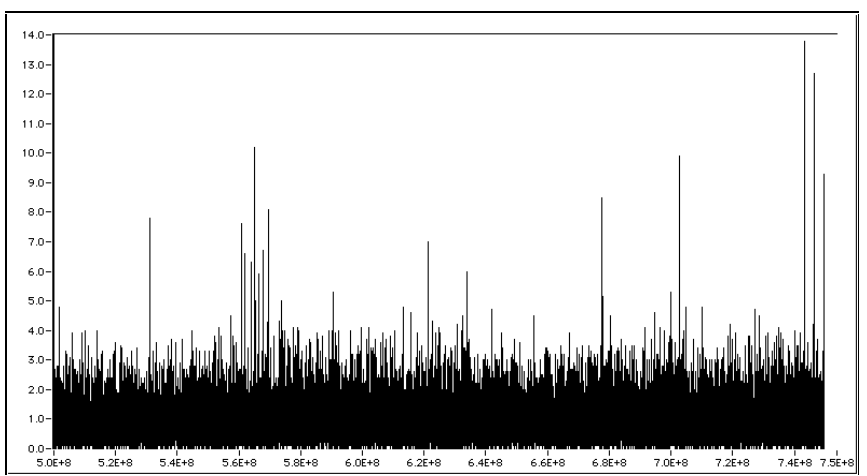

Figure 4. Difference in Beam Spectra between Cavity at Nominal Operating Temperature and $45^{\circ} \mathrm{C}$.

Fig. 4 shows the difference in beam spectra when the cavity temperature was moved to $45^{\circ} \mathrm{C}$. The span of Fig's. 4 and 5 is $250 \mathrm{MHz}$ with a resolution bandwidth on the HP8568B High Frequency Spectrum Analyser of $3 \mathrm{KHz}$.

The differences that appear in Fig. 4 are isolated orbit harmonics about the $499.71 \mathrm{MHz}$ RF source signal with an amplitude $<15 \mathrm{~dB}$. These difference peaks are very close to the noise floor in this sort of measurement and are considered to be negligible.

When one looks at the beam spectra difference when the cavity temperature is at $47^{\circ} \mathrm{C}$, then large differences are observed at $608.96 \mathrm{MHz}$ and orbit harmonics about this peak (see Fig.5).

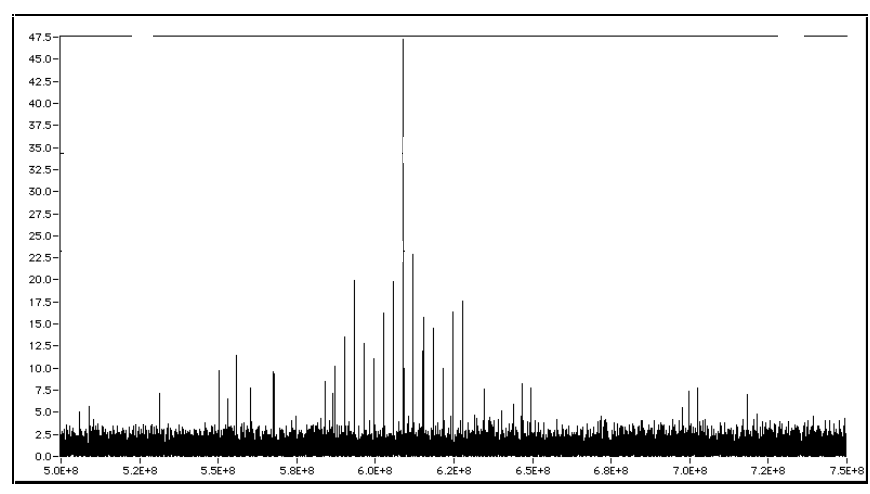

Figure 5. Difference in Beam Spectra between Cavity at Nominal Operating Temperature and $47^{\circ} \mathrm{C}$.

The difference is clear and it shows that the instability observed on the photon beam appears to be as a result of this resonance. The oscillatory behaviour of the photon beam position is not yet fully understood as some oscillation is still present when the cavity temperature is brought back to its normal operating temperature, investigations are ongoing.

\section{MAFIA PREDICTED CAVITY HOM's..}

MAFIA simulations of the SRS cavity have been used to provide HOM information as well as predicting e-m field orientations of these HOM's which can lead to an understanding of their interaction on the electron beam, see Table 1 [2]. Relating these HOM's to the results of Fig. 5, shows that one mode in particular could be reponsible for the observed instability. The $1390 \mathrm{MHz}$ HOM, which when wrapped around the $499.711 \mathrm{MHz}$ fundamental RF frequency and its harmonics, produce resonances at $1610 \mathrm{MHz}$, $1110 \mathrm{MHz}, 890 \mathrm{MHz}$ and $610 \mathrm{MHz}$.

\begin{tabular}{|c|c|c|}
\hline Mode & $\begin{array}{c}\text { Frequency } \\
\text { (MHz) }\end{array}$ & $\boldsymbol{R}_{\boldsymbol{s}}^{\mathbf{1}}(\mathbf{M} \boldsymbol{\Omega})$ \\
\hline Mono & 498.8 & 3.95 \\
\hline Dip (V) & 791.5 & 6.2 \\
\hline Dip (H) & 797.1 & 7.2 \\
\hline Mono & 809.5 & 1.15 \\
\hline Dip (V) & 1059.2 & 12.3 \\
\hline Dip (H) & 1059.3 & 12.3 \\
\hline Dip (V) & 1285.0 & 1.85 \\
\hline Dip (H) & 1286.0 & 1.9 \\
\hline Mono & 1333.0 & 0.4 \\
\hline Dip & 1390.0 & $\sim 0.9$ \\
\hline
\end{tabular}

Table 1. SRS Cavity HOM's as Predicted by MAFIA.

1 Shunt Impedance determination assumes r.m.s. voltage i.e. $R_{s}=\frac{V^{2}}{2 P} \quad$ where: $\quad P=$ Cavity Power Dissipation (W) 
MAFIA has found that some dipole modes are split into two polarisations, with a null plane in the longitudinal electric field. Their action on the electron beam will be to deflect it horizontally or vertically, hence the $\mathrm{H}$ and $\mathrm{V}$ denotation.

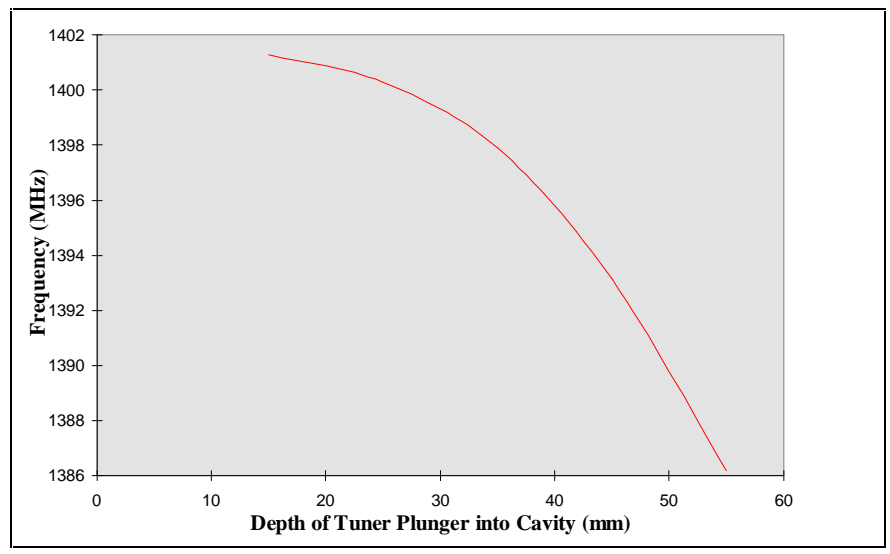

Figure 6. Frequency Variation of $1390 \mathrm{MHz} \mathrm{HOM}$ as a Function of Tuner Position.

When one examines the shift in frequency of the $1390 \mathrm{MHz}$ mode, as a function of tuner position, the range over which the tuner operates on the actual cavity causes quite large frequency variations. Which gives this particular HOM a large operating frequency range.

The e-m field orientations of this mode are complex and its action on the electron beam is not fully understood. The mode does contain some skew dipole action and it is this property that is believed to be causing the electron beam movements.

The SRS operates with an orbit harmonic of $3.123 \mathrm{MHz}$ and the 286th orbit harmonic coincides with this HOM.

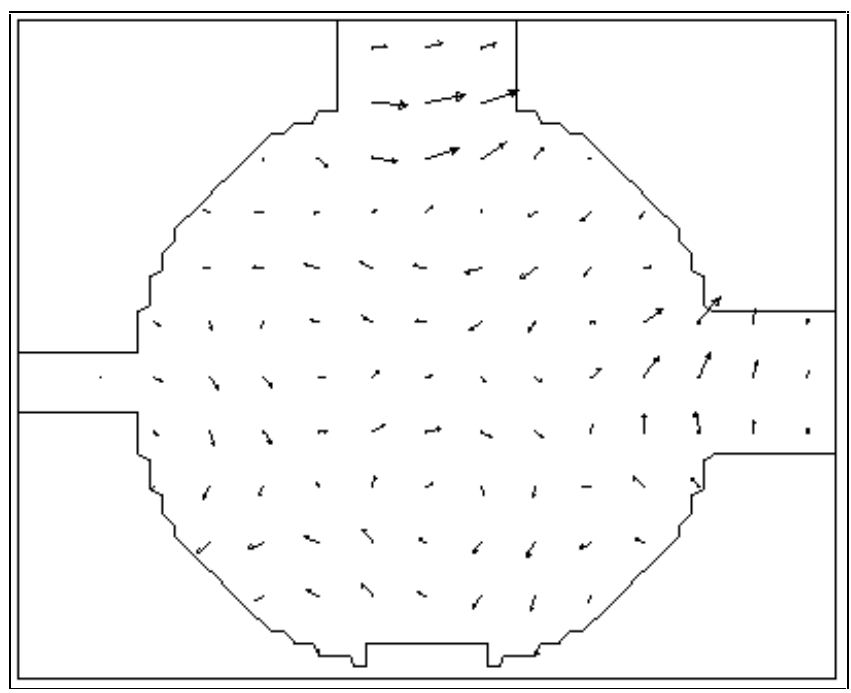

Figure 7. E field Orientation of 1390MHz HOM.

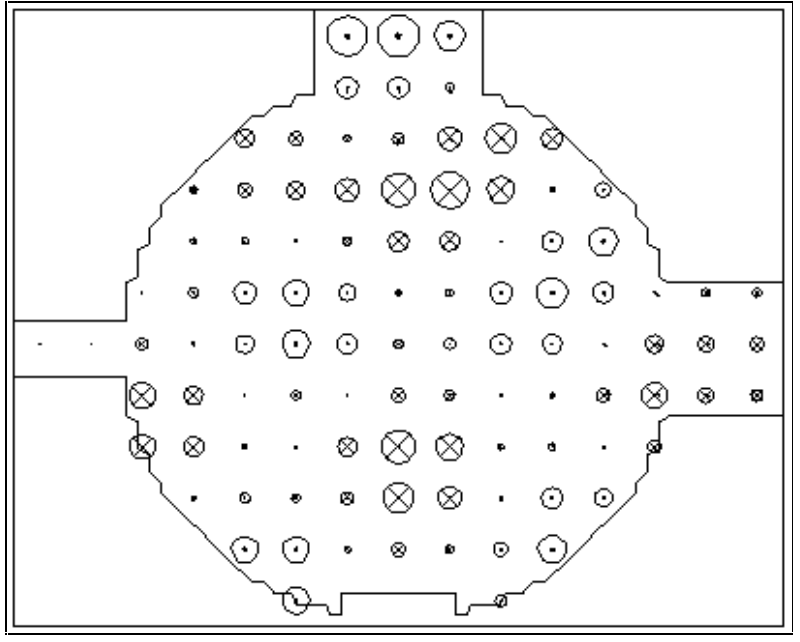

Figure 8. H field Orientation of 1390MHz HOM.

\section{CONCLUSIONS.}

Under normal operating conditions, the cavity temperature is chosen such that the normal range of tuner plunger position does not enable the beam to excite any dangerous HOM's. The experiment varied the cavity temperature by $\pm 2^{\circ} \mathrm{C}$ and clearly the $1390 \mathrm{MHz}$ mode was excited. The beam movements were observed on all TVM's and BPM's.

It is clear that a malfunction in the cavity water cooling system causing the cavity temperature to vary outside the specified limits $\left( \pm 0.2^{\circ} \mathrm{C}\right)$, will cause the position of the cavity tuner plunger to vary. The detailed HOM spectra is dependent on cavity tuner plunger position.

The fault was quickly rectified and since new higher precision water temperature controllers have been fitted to all cavity water cooling circuits, the temperatures are now maintained to better than $\pm 0.1^{\circ} \mathrm{C}$.

\section{REFERENCES}

[1] Second Annual Workshop on Synchrotron Radiation Light Sources, Grenoble 15th and 16th November 1994, by: M. Svandrlik, ELETTRA.

[2] Higher Order Modes in the SRS $500 \mathrm{MHz}$ Accelerating Cavities, J. N. Corlett, presented at the 1989 Particle Accelerator Conference on Accelerator Science and Technology, Chicago. DL/SCI/P627A 